Case Report

\title{
PROSTHODONTIC REHABILITATION OF A PATIENT WITH ORAL SUBM UCOUS FIBROSIS AND MICROSTOMIA : A CASE REPORT
}

\author{
Anupama Prasad D. ${ }^{1}$, Krishna Prasad D. ${ }^{2}$, Chethan Hegde. ${ }^{3}$ \\ ${ }^{1}$ Lecturer, ${ }^{2}$ Professor and H.O.D., ${ }^{3}$ Professor, Department of Prosthodontics and Crown \& Bridge, \\ A.B. Shetty Memorial Institute of Dental Sciences, Nitte University, Deralakatte , Mangalore - 575018 \\ Correspondence : \\ Anupama Prasad D. \\ Lecturer, Department of Prosthodontics, A.B. Shetty M emorial Institute of Dental Sciences, Nitte University, \\ Deralakatte , Mangalore - 575 018, E-mail : anupamaprasadd@ymail.com
}

\begin{abstract}
:
This case report deals with treatment of a female patient aged 45 years with limited mouth opening due to submucous fibrosis, who was completely edentulous and was prosthetically treated with modification of techniques for impression making and jaw relation so that a complete denture was made without sections which could be easily inserted and removed without compromising retention and esthetics.
\end{abstract}

Keywords: limited mouth opening, oral submucous fibrosis, sectional trays, microstomia

\section{Introduction:}

Oral submucous fibrosis, an insidious chronic disease and a precancerous condition, affecting any part of the oral cavity and sometimes the pharynx is caused by prolonged use of tobacco, arecanut, spices ,etc., Schwartz in $1952^{1}$ described a condition of the oral mucosa which included the palate and pillars of the fauces, and called it"atrophia idiopathica (tropica) mucosae oris". It was later termed oral submucous fibrosis. Various synonyms are "diffuse oral submucous fibrosis" by Joshi in 1953, "idiopathic scleroderma of the mouth", "idiopathic palatal fibrosis", "sclerosing stomatitis" and "juxta-epithelial fibrosis" .Gupta et al., ${ }^{3}$ reported malignant transformation in $2.3 \%$ of patients with Oral Submucous Fibrosis from a 10-year follow-up study in Ernakulam district, Kerala. It is often associated with limited mouth opening due to increased fibrosis.This disease is characterized by burning sensation of the mouth, stiffening of certain areas of oral mucosa with difficulty in opening the mouth. Oral submucous fibrosis, now globally accepted as an Indian disease has one of the highest rates of malignant transformation. Mucosa eventually becomes blanch and opaque and fibrotic bands appear involving buccal mucosa, soft palate, lips and tongue. Oral epitheleum is usually atrophied with complete loss of rete pegs, hyalinization of the connective tissue, homogenization of collagen bundles and obliteration of blood vessel. The fibrosis involves the lamina propria and the submucosa and may often extend into the underlying musculature resulting in the deposition of dense fibrous bands giving rise to the limited mouth opening which is a hallmark of this disorder ${ }^{4}$.

Microstomia is defined as an abnormally small oral orifice. Other causes of microstomia are scleroderma, oral submucous fibrosis, sequale of burns, surgical resection of facial and oral neoplasms and temporo mandibular joint disorders. Prosthetic rehabilitation of patients with oral submucous fibrosis and microstomia presents difficulties at all stages, from the preliminary impressions to fabrication of prosthesis. Since they have a small oral opening, it may be impossible to make impressions and fabricate complete dentures using conventional methods. As the size of the oral opening decreases, the difficulty in the planned treatment procedures also increases.

\section{Case report:}

A female patient from Kerala state aged 45 years with complete edentulous arches, reported to the department of Prosthodontics, for prosthodontic rehabilitation. On clinical examination, the patient was found to have oral submucous fibrosis with microstomia. Patient also gave a prolonged history of 25 years of arecanut / beetlenut chewing. On examination, maximum mouth opening of the patient was restricted to $20 \mathrm{~mm}$. Intercommisural width was $18 \mathrm{~mm}$ (figure 1 ). On palpation, the mucous membrane was firm and there was a fibrous band extending from the buccal aspect of molar area upto the angle of mouth, with 
fibrotic bands connecting the lower lip unilaterally to the residual ridge .The patient had been treated for limited mouth opening with intralesional injections the detail of which the patient was unaware of.

Since mouth opening was limited, the smallest available stock tray (size-0) was selected whose flanges were shortened and the tray was bent with help of pliers until it could be inserted in patients mouth and the impression was made with irreversible hydrocolloid (Neocolloid, Zermack clinical, Italy) as the impression material. Once the cast (type III stone Kalabhai, M umbai, India) was poured, it was decided that a sectional special tray would be fabricated for convenience of recording the final impression accurately. Wax spacers(Deepthi, modelling wax, India), were then adapted such that there were four tissue stops in each section to aid in stabilizing the tray when used in sections in maxillary arch , while mandibular tray was in a single piece.

A sectional impression tray was designed with right and left sections that could be detached and then joined together in the correct original position with the help of acrylic resin blocks which were joined with help of snap fit pins ${ }^{5,6}$. Maxillary and mandibular impression trays were then fabricated (figure 2). Autopolymerizing acrylic resin (Dentsply, Gurgaon, India) was used for the fabrication of the trays. Border molding of each half of the arches were carried out seperatly(figure 3 ) with green stick compound (DPI Pinnacle Tracing sticks, Bombay, India) followed by making of final impression which were then joined extraorally to form a single unit. Final impressions were made with non-Eugenol impression material as Eugenol would cause burning sensation to the patient. The mandibular impression was carried out in a single tray.

Inserting the occlusion rim also would pose a problem for which the denture base itself was fabricated in sections(figure 4), on which a sectional occlusion rim (figure 5)was made. During the stage of trial of the waxed up dentures, we noted that by altering the path of placement of the maxillary complete denture with a more horizontal path of placement it was possible to place the denture in one piece which eliminated the need for construction of a sectional denture which though would be convenient for placement, would be difficult in maintenance of hygiene. The maxillary and mandibular complete dentures(figure 6) were then acrylized conventionally in heat polymerizing acrylic resin (Trevelon, Dentsply, Gurgaon, India).

\section{Discussion :}

Microstomia or limited mouth opening is common in patients suffering from oral submucous fibrosis. This condition poses problem during each step of prosthetic reconstruction. Several methods of constructing sectional special trays have been discussed in literatures. The plastic tray can be cut in two sections with a disc with the handle in the larger section. Three building blocks(toy) were selected to re-approximate sectional trays by Leuboke ${ }^{6,7}$. A technique of pouring impression plaster onto the tissue surface of the patient's previous denture (constructed before microstomia condition set in) and a cast obtained on which special tray can be constructed was given by J.Fraser, Mc Cord et al., ${ }^{6,8}$.Putty silicone was also used as flexible tray ${ }^{9}$. In the present case we used a simple method wherein acrylic blocks snap fit buttons and acrylic blocks were used and stabilized and jaw relation was also carried out with divided denture base but the final denture was not sectional, as maintenance of oral hygiene was of prime concern because the patient had xerostomia due to oral submucous fibrosis and there was high risk of oral infections like candidiasis for the same reason.

\section{Summary and Conclusion :}

- In our treatment plan we selected a very economical method for making special tray with the help of snap fit buttons and acrylic blocks which are available easily anywhere and also would not increase the height of the tray, while the acrylic blocks stabilized the tray during the pouring of gypsum product into the tray.

- These pins and blocks could be eliminated by slight trimming during the stage of acrylization from the denture base also.

The mandibular left posterior region provided space for the placement of teeth only till the second premolars, but in order to maintain symmetry and also to retain the denture in place, teeth were arranged bilaterally with one premolar and one molar by eliminating the second premolars and second molars.

Only prosthetic rehabilitation will not suffice in such cases, 
it should be supported with cessation of arecanut eating, salivary substitutes, nutritional support high in protein and calories and for vitamin B complex and other vitamins and minerals., physiotherapy with heat therapy like hot rinses, lukewarm water, or selective deep heating therapies like short-wave and microwave diathermy .

\section{Figures:}

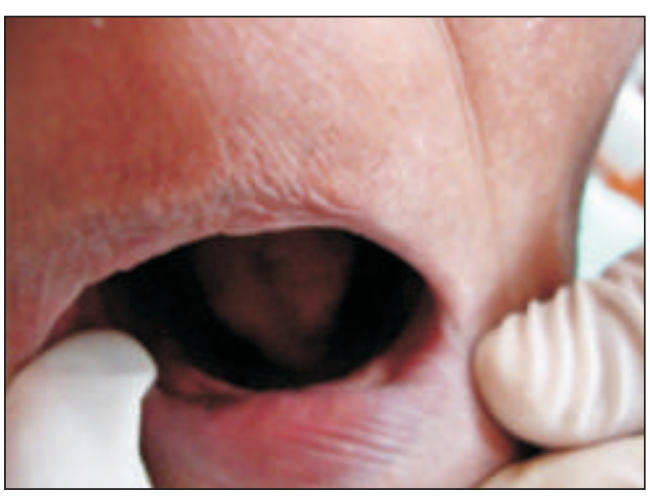

Fig 1: Limited mouth opening

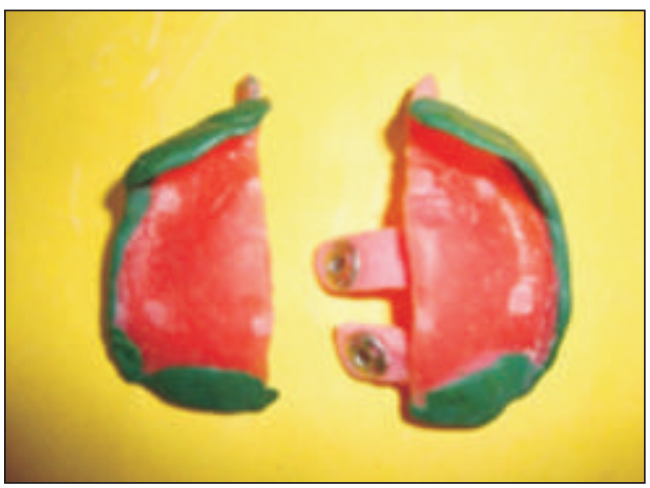

Fig 3: Border molding in sections

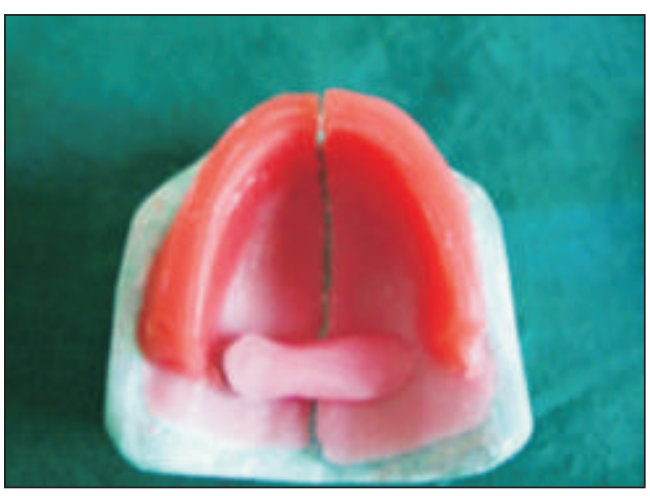

Fig 5: occlusion rims in two sections
A treatment provided for a compromised patient with which there will be a change in appearance and function would be one of the best gifts to her as she will develop self confidence and the social status would improve a lot and the patient will be ever grateful for the treatment given. No condition is impossible to treat if slight modifications can be thought of in the methods of treatment.

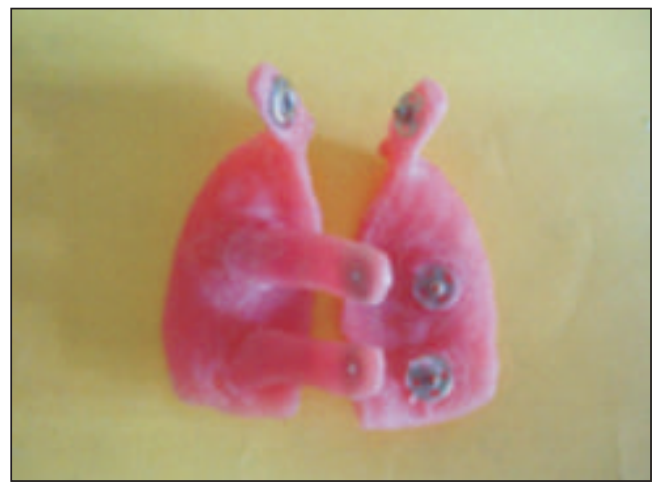

Fig 2: Sectional impression tray with snap fit pins

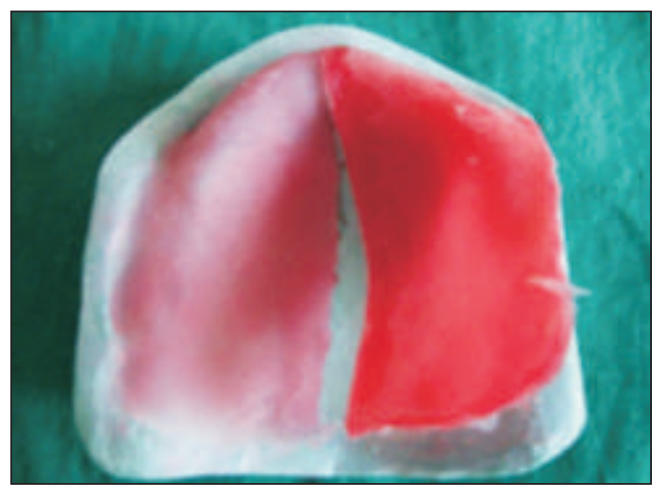

Fig 4: Denture base in two halves

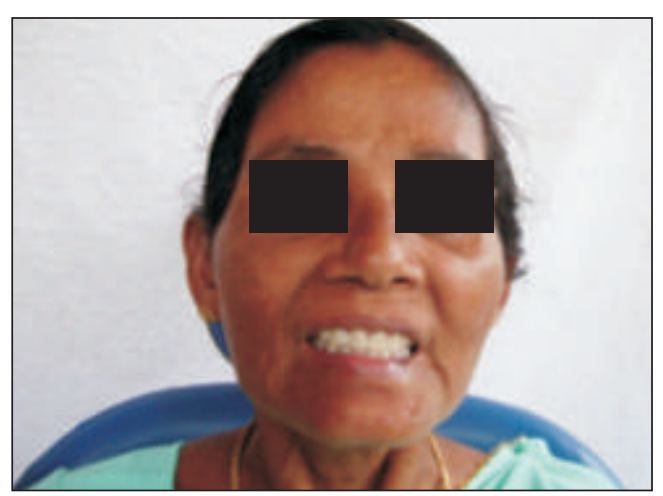

Fig 6: Post treatment view 


\section{References:}

1. Rajendran R. Oral submucous fibrosis: etiology, pathogenesis, and future research. Bulletin of the World Health Organization, 1994; 72 (6): 985-96.

2. Pindborg JJ, Sirsat SM. Oral submucous fibrosis. Oral surg. oral med. oral pathol. 1966; 22:764-79.

3. Gupta PC et al.,. Incidence rates of oral cancer and natural history of oral pre-cancerous lesions in a 10-year follow-up study of Indian villagers. Community dent. oral epidemiol.1980; 8: 287-333.

4. Gupta M.K., M haske S, Ragavendra R, Imtiyaz. Oral submucous fibrosis - Current Concepts in Etiopathogenesis. People's Journal of Scientific Research 2008;1:39-44.

5. Prasad R, Bhide SV, Gandhi PV, Divekar NS, M adhavVNV. Prosthodontic management of a patient with limited mouth opening: A practical approach. JIPS2008; 8:83-6.

6. Impression Tray Designs and Techniques for Complete Dentures in Cases of Microstomia - A Review. Hegde C, Prasad K, Prasad A, Hegde R.J Prosthodont Res 2011[Epub ahead of print]

7. Luebke RJ. Sectional impression tray for patients with constricted oral opening.J Prosthet Dent 1984;52:135-7.

8. J.Fraser M c Cord, Kenneth W.Tyson, Ian S.Blair. A sectional complete denture for a patient with microstomia. J Prosthet Dent 1989;61:6457.

9. Whisitt JA, Battle LW. Technique for making flexible impression trays for the microstomic patient. J Prosthet Dent 1984;52:608-9. 